\title{
Tracing a high redshift cosmic web with quasar systems $\mathbf{s}^{\star} \star \star$
}

\author{
Maret Einasto $^{1}$, Erik Tago ${ }^{1}$, Heidi Lietzen ${ }^{1,2,3}$, Changbom Park ${ }^{4}$, Pekka Heinämäki ${ }^{5}$, Enn Saar ${ }^{1,6}$, Hyunmi Song ${ }^{4}$, \\ Lauri Juhan Liivamägi ${ }^{1,7}$, and Jaan Einasto ${ }^{1,6,8}$ \\ 1 Tartu Observatory, 61602 Tõravere, Estonia \\ e-mail: maret@aai.ee \\ 2 Instituto de Astrofísica de Canarias, 38205 La Laguna, Tenerife, Spain \\ 3 Universidad de La Laguna, Dept. Astrofísica, 38206 La Laguna, Tenerife, Spain \\ ${ }^{4}$ School of Physics, Korea Institute for Advanced Study, 85 Hoegiro, Dong-Dae-Mun-Gu, 130-722 Seoul, Korea \\ 5 Tuorla Observatory, University of Turku, Väisäläntie 20, Piikkiö, Finland \\ ${ }^{6}$ Estonian Academy of Sciences, 10130 Tallinn, Estonia \\ 7 Institute of Physics, Tartu University, Tähe 4, 51010 Tartu, Estonia \\ ${ }^{8}$ ICRANet, Piazza della Repubblica 10, 65122 Pescara, Italy
}

Received 27 May 2014 / Accepted 20 June 2014

\section{ABSTRACT}

Context. To understand the formation, evolution, and present-day properties of the cosmic web we need to study it at low and high redshifts.

Aims. We trace the cosmic web at redshifts that range from $1.0 \leq z \leq 1.8$ by using the quasar (QSO) data from the SDSS DR7 QSO catalogue.

Methods. We apply a friend-of-friend algorithm to the quasar and random catalogues to determine systems at a series of linking length and analyse richness and sizes of these systems.

Results. At the linking lengths $l \leq 30 h^{-1} \mathrm{Mpc}$, the number of quasar systems is larger than the number of systems detected in random catalogues, and the systems themselves have smaller diameters than random systems. The diameters of quasar systems are comparable to the sizes of poor galaxy superclusters in the local Universe. The richest quasar systems have four members. The mean space density of quasar systems, $\approx 10^{-7}\left(h^{-1} \mathrm{Mpc}\right)^{-3}$, is close to the mean space density of local rich superclusters. At intermediate linking lengths $\left(40 \leq l \leq 70 h^{-1} \mathrm{Mpc}\right)$, the richness and length of quasar systems are similar to those derived from random catalogues. Quasar system diameters are similar to the sizes of rich superclusters and supercluster chains in the local Universe. The percolating system, which penetrate the whole sample volume appears in a quasar sample at a smaller linking length than in random samples (85 $\left.\mathrm{h}^{-1} \mathrm{Mpc}\right)$. At the linking length $70 \mathrm{~h}^{-1} \mathrm{Mpc}$, the richest systems of quasars have diameters exceeding $500 \mathrm{~h}^{-1} \mathrm{Mpc}$. Quasar luminosities in systems are not correlated with the system richness.

Conclusions. Quasar system catalogues in our web pages and at the Strasbourg Astronomical Data Center (CDS) serve as a database for searching superclusters of galaxies and for tracing the cosmic web at high redshifts.

Key words. large-scale structure of Universe - quasars: general

\section{Introduction}

The contemporary cosmological paradigm tells us that the structure in the Universe, the cosmic web formed and evolved from tiny density perturbations in the very early Universe by hierarchical growth, is driven by gravity (see, e.g., Loeb 2008; van de Weygaert \& Schaap 2009, and references therein). Groups and clusters of galaxies and their filaments are formed by density perturbations on a scale up to $32 \mathrm{~h}^{-1} \mathrm{Mpc}$. Superclusters of galaxies, the largest density enhancements in the local cosmic web, are formed by larger-scale perturbations up to $100 \mathrm{~h}^{-1} \mathrm{Mpc}$. Still, larger scale density perturbations modulate the richness of galaxy systems (Einasto et al. 2011a; Suhhonenko et al. 2011).

To understand how the cosmic web is formed and evolved, it is of paramount importance to describe and quantify it at low and high redshifts. Large galaxy redshift surveys like SDSS enable

* Appendix A is available in electronic form at http://www . aanda.org

$\star \star$ The catalogues are only available at the CDS via anonymous ftp to cdsarc.u-strasbg.fr (130.79.128.5) or via

http://cdsarc.u-strasbg.fr/viz-bin/qcat?]/A+A/568/A46 us to describe the cosmic web in our neighbourhood in detail. One source of information about the cosmic structures at high redshifts is the distribution of quasars. Quasars lie at centres of massive galaxies (Hutchings et al. 2002; Kotilainen et al. 2009; Padmanabhan et al. 2009; Letawe et al. 2010; Kotilainen et al. 2013; Falomo et al. 2013; Floyd et al. 2013; Falomo et al. 2014; Karhunen et al. 2014, and references therein), which typically lie in small groups and poor clusters of galaxies (Wold et al. 2000; Soechting et al. 2002; Söchting et al. 2004; Coldwell \& Lambas 2006; Hutchings et al. 2009; Trainor \& Steidel 2012; Ueda et al. 2013). Nearby quasars are typically located in a relatively lowdensity, large-scale environment near superclusters of galaxies (Coldwell \& Lambas 2006; Lietzen et al. 2009, 2011).

Galaxy luminosities, morphological types, colours, star formation rates, and other properties are closely related to their environment at small and large scales (Einasto et al. 1974; Dressler 1980; Einasto \& Einasto 1987; Mo et al. 1992; Park et al. 2007; Park \& Choi 2009; Skibba 2009; Tempel et al. 2011; Lietzen et al. 2012; Einasto et al. 2014). The studies of the quasar environment in the cosmic web helps us to understand the relation between the galaxy and quasar evolution and to test the evolutionary schemes of different type of objects. 
Already decades ago, several studies described large systems in quasar distribution (Webster 1982; Clowes \& Campusano 1991; Komberg et al. 1996; Williger et al. 2002; Clowes et al. 2012, 2013, and references therein), which are known as large quasar groups (LQGs). Miller et al. (2004) found significant $200 \mathrm{~h}^{-1} \mathrm{Mpc}$ size over- and underdensities in the density field determined by quasars from the $2 \mathrm{dF}$ QSO Redshift survey. Komberg et al. (1996) showed that LQGs may trace distant galaxy superclusters. The large-scale distribution of quasar systems gives us information about the cosmic web at high redshifts which are not yet covered by large and wide galaxy surveys.

The goal of this study is to analyse the distribution of quasars at redshifts in the range of $1.0 \leq z \leq 1.8$. We choose this redshift range as we are interested in the study of the cosmic web at high redshifts. This redshift range was also used by Clowes et al. (2012, 2013); with this choice, we can compare LQGs found in these studies and our systems. We shall use the Schneider et al. (2010) catalogue of quasars based on the Sloan Digital Sky Survey Data Release 7 (SDSS DR7). We analyse clustering properties of quasars with the friend-of-friend (FoF) algorithm, compare them with random distributions, and determine systems of quasars at a series of linking lengths. We present catalogues of quasar systems and analyse their properties and large-scale distribution.

In Sect. 2 we describe the data we used and the method in Sect. 3. In Sect. 4, we give the results. We discuss and summarise the results in Sect. 5. In Appendix A, we present data about the richest quasar systems.

We present the quasar system catalogues ${ }^{1}$. An interactive pdf file showing the distribution of quasars is also available on our web pages.

We assume the standard cosmological parameters: the Hubble parameter $H_{0}=100 \mathrm{hm} \mathrm{s}^{-1} \mathrm{Mpc}^{-1}$, the matter density $\Omega_{\mathrm{m}}=0.27$, and the dark energy density $\Omega_{\Lambda}=0.73$.

\section{Data}

We adopt a catalogue of quasars with a spectrum taken as a normal science spectrum (SCIENCEPRIMARY=1) and a photometric measurement that is designated PRIMARY in the BEST photometric database. From this catalogue, we select a subsample of quasars in the redshift interval $1.0 \leq z \leq 1.8$ and apply an $i$-magnitude limit $i=19.1$. The full quasar catalogue by Schneider et al. (2010) is not homogeneous; Vanden Berk et al. (2005) and Richards et al. (2006) (see also Clowes et al. 2012) gave a detailed analysis of the completeness of the sample and suggested to use these selection limits for statistical studies. To reduce the edge effects of our analysis, we limit the data in the area of SDSS sky coordinate limits $-55 \leq \lambda \leq 55$ degrees and $-33 \leq \eta \leq 35$ degrees. Our final sample contains data of 22381 quasars.

In Fig. 1, we plot the number density of quasars at different distances. The mean space density of quasars is approximately $1.1 \times 10^{-6}\left(h^{-1} \mathrm{Mpc}\right)^{-3}$. The density decreases slightly with distance. Below, we analyse whether this trend affects the result of our analysis. Figure 1 demonstrates several wiggles in the number density of quasars, which correspond to the location of very rich quasar systems, as we show below.

The space density of quasars is very low; therefore, it is important to understand whether their distribution differs from random distribution. To compare quasar and random distributions, we generated random samples using the same coordinate $\lambda$

\footnotetext{
1 http://www . aai.ee/ maret/QSOsystems.html
}

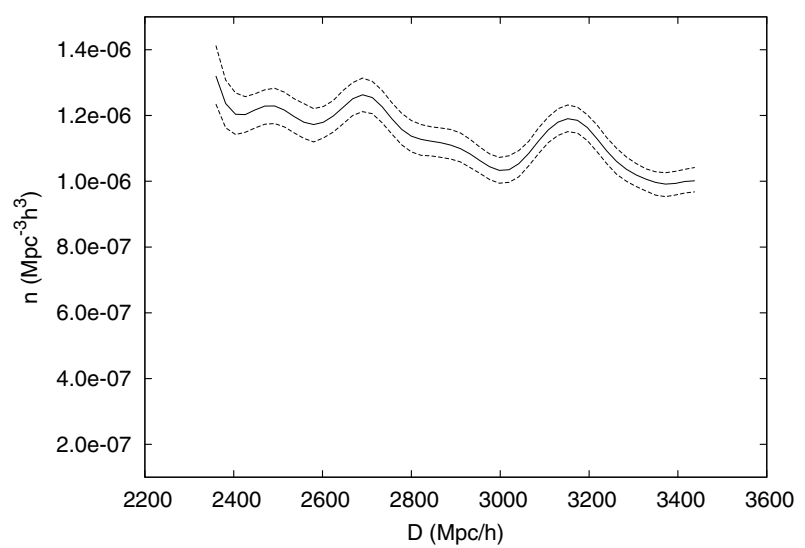

Fig. 1. Quasar number density versus distance. Dotted lines show the $98 \%$ central confidence limits.

and $\eta$, and redshift limits as quasar samples. The number of random points in random samples was equal to the number of quasars.

\section{Method}

We employed the FoF clustering analysis method introduced in cosmology by Zeldovich et al. (1982) and Huchra \& Geller (1982) to determine systems in the quasar catalogue and random catalogues. The FoF method is commonly used to detect groups and clusters in the galaxy distribution (Tucker et al. 2000; Eke et al. 2004; Tago et al. 2006, 2008; Park et al. 2012; Tempel et al. 2012, 2014, and references therein) and superclusters of optical and X-ray clusters of galaxies (Einasto et al. 1994, 2001; Chon et al. 2013). Komberg et al. (1996) applied the FoF method to search for rich quasar systems.

The FoF method collects objects into systems if they have at least one common neighbour closer than a linking length. At small linking lengths where only the closest neighbouring objects form systems, most objects in the sample remain single. As the linking length increases, more and more objects join systems, and the number of systems, their richness and size increase. At a certain linking length, the largest system spans over the whole sample volume and a percolation occurs. We apply the FoF algorithm to quasar and random samples with a series of linking lengths to analyse the properties of systems and their percolation. Klypin \& Shandarin (1993) showed that the richness and size of the richest and the second richest system in a sample characterise the growth of systems and percolation properties of a sample well. Therefore, we analyse the properties of the richest systems in quasar and random catalogues at each linking length.

\section{Results}

\subsection{FoF analysis}

The FoF algorithm was applied to the observed quasar and random catalogues at a series of linking lengths. At each linking length, we found the number of systems in quasar and random samples with at least two members, calculated multiplicity functions of systems, and found the length and size of the first and second largest system. In Tables A.1-A.3, we present the data on the richest systems of quasars at linking lengths 20,30, and $70 h^{-1}$ Mpc.

Figure 2 shows the number of systems from the quasar and random catalogues (upper panel) and the ratio of the numbers 

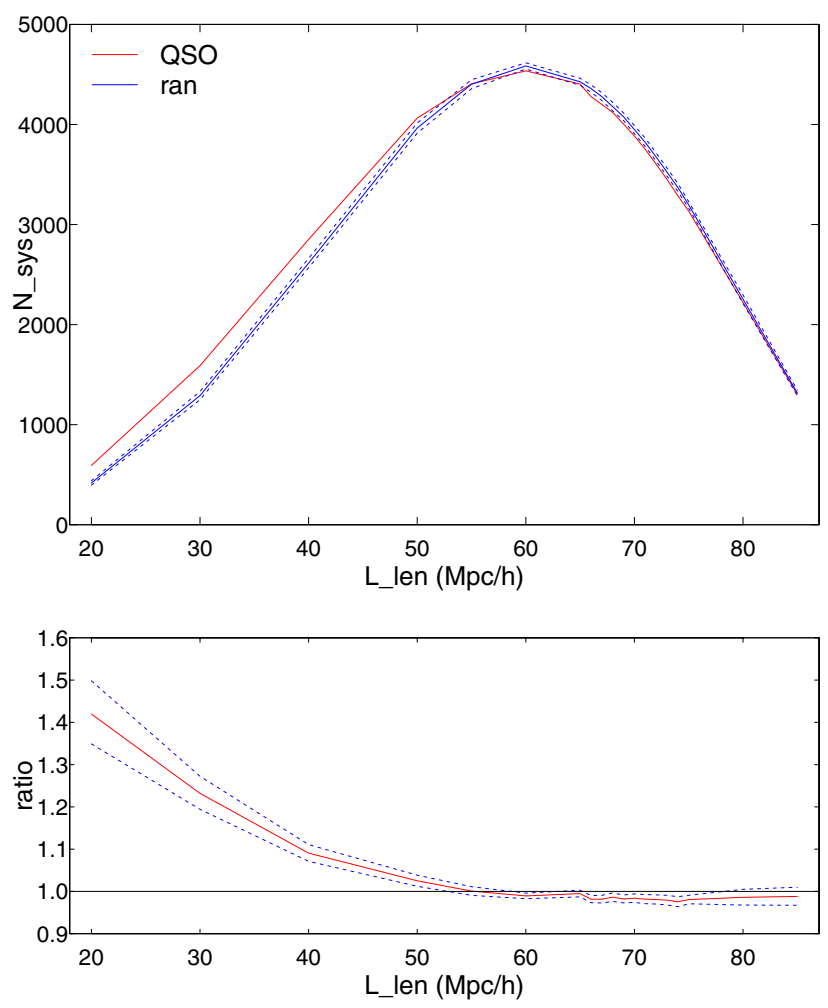

Fig. 2. Number of quasar and random systems (upper panel) and ratio of the numbers of quasar and random systems (lower panel) vs. the linking length. Red solid lines denote quasar systems, and blue lines denote random systems, black line shows ratio 1 .

of systems (lower panel). At the linking lengths up to approximately $50 h^{-1} \mathrm{Mpc}$, the number of quasar systems is larger than the number of systems in random catalogues. At higher values of the linking lengths the number of systems in the quasar catalogue becomes similar to that in random catalogues. The number of systems in both quasar and random catalogues reach maximum at $60 h^{-1} \mathrm{Mpc}$. At higher values of the linking lengths, systems begin to join into larger systems and the number of systems decreases.

The richness and diameters (maximum distance between quasar pairs in a system) of the largest two systems in quasar and random catalogues is given in Table 1. At the linking lengths $l \leq 30 h^{-1} \mathrm{Mpc}$, the richest quasar systems have smaller diameters than the richest random systems. In a wide linking length interval, $40 \leq l \leq 70 h^{-1} \mathrm{Mpc}$, the richness and diameters of the richest quasar systems are comparable to those found in random catalogues. At the linking length $80 \mathrm{~h}^{-1} \mathrm{Mpc}$, quasar systems start to join; the richness and diameter of the richest quasar system increase rapidly. The increase of the richness and size of the richest random system is much smaller. At the linking length $l=85 h^{-1} \mathrm{Mpc}$ about half of quasars join the richest quasar system, and a percolation occurs. The richness and diameter of the richest system in random catalogues also increase, but this system is still poorer and smaller than the richest system in the quasar catalogue. The second richest system in random catalogues is much richer than the second richest system in the quasar catalogue. This shows that the percolation of quasar and random systems occurs differently, and the properties of the richest systems differ.

We also analysed the multiplicity functions, which show the fractions of systems of different richness in quasar and random samples at a series of linking lengths. Up to the linking length
Table 1. Number of objects in the two richest systems, and the system diameters from quasar and random catalogues at a series of linking lengths.

\begin{tabular}{rrcrc}
\hline \hline$(1)$ & $(2)$ & $(3)$ & $(4)$ & $(5)$ \\
$l$ & $\mathrm{~N} 1_{\mathrm{QSO}}$ & $\mathrm{N} 1_{\text {ran }}$ & $\mathrm{D} 1_{\mathrm{QSO}}$ & $\mathrm{D} 1_{\text {ran }}$ \\
\hline 20 & 3 & $3.3 \pm 0.5$ & 31.0 & $33.5 \pm 2.0$ \\
30 & 4 & $4.9 \pm 0.8$ & 58.8 & $70.0 \pm 5.2$ \\
40 & 7 & $7.6 \pm 1.3$ & 109.4 & $119.2 \pm 17.4$ \\
50 & 15 & $12.3 \pm 2.2$ & 178.6 & $209.7 \pm 14.7$ \\
60 & 23 & $21.3 \pm 2.0$ & 324.9 & $369.8 \pm 40.1$ \\
70 & 67 & $65.1 \pm 11.7$ & 703.1 & $717.1 \pm 86.0$ \\
75 & 105 & $146.3 \pm 39.9$ & 865.3 & $992.5 \pm 145.9$ \\
80 & 920 & $460.0 \pm 54.1$ & 2066.4 & $1750.2 \pm 171.1$ \\
85 & 11415 & $6464.0 \pm 1886.3$ & 5060.3 & $4552.7 \pm 645.4$ \\
\hline$l$ & $\mathrm{~N} 2 \mathrm{QSO}$ & $\mathrm{N} 2_{\text {ran }}$ & $\mathrm{D} 2 \mathrm{QSO}$ & $\mathrm{D} 2_{\text {ran }}$ \\
\hline 20 & 3 & $2.3 \pm 0.5$ & 29.9 & $30.9 \pm 2.6$ \\
30 & 4 & $3.7 \pm 0.5$ & 49.4 & $61.4 \pm 3.6$ \\
40 & 7 & $6.0 \pm 0.9$ & 106.8 & $104.3 \pm 4.9$ \\
50 & 14 & $10.6 \pm 1.5$ & 177.7 & $185.6 \pm 16.3$ \\
60 & 20 & $19.9 \pm 1.9$ & 294.0 & $307.0 \pm 29.3$ \\
70 & 64 & $56.3 \pm 7.8$ & 614.6 & $626.2 \pm 46.9$ \\
75 & 97 & $109.1 \pm 24.9$ & 792.3 & $883.5 \pm 53.8$ \\
80 & 421 & $372.4 \pm 71.3$ & 1832.7 & $1539.5 \pm 143.0$ \\
85 & 678 & $2652.3 \pm 859.1$ & 1789.1 & $2337.6 \pm 782.2$ \\
\hline
\end{tabular}

Notes. Columns are as follows: (1) linking length $l$ in $h^{-1} \mathrm{Mpc}$; (2) the number of quasars in the 1 st and 2 nd richest quasar system; (3) mean number of random points in the 1st and 2nd richest quasar system; (4) the diameter of the 1 st and the 2 nd richest quasar system in $h^{-1} \mathrm{Mpc}$; (5) the diameter of the 1 st and the 2 nd richest random system in $h^{-1} \mathrm{Mpc}$.

$l=70 h^{-1} \mathrm{Mpc}$, the multiplicity functions for quasar and random catalogues are very similar. At the linking length $80 \mathrm{~h}^{-1} \mathrm{Mpc}$, the richness of the richest quasar system increases rapidly, and systems start to join into one huge percolating system, as shown above, and multiplicity functions of quasars and random systems become different. The differences are the largest at the linking length $85 h^{-1} \mathrm{Mpc}$.

Next, we compare the distribution of diameters of quasar and random systems. In Fig. 3 we show the numbers of quasar and random systems with different diameters at the linking lengths of 30 and $70 h^{-1} \mathrm{Mpc}$. At the linking length $20 h^{-1} \mathrm{Mpc}$, the diameters of the triple quasar systems are given in Table A.1.

At the linking lengths of 20 and $30 \mathrm{~h}^{-1} \mathrm{Mpc}$, most systems in both quasar and random catalogues are pairs, the richest systems have four (three at $l=20 h^{-1} \mathrm{Mpc}$ ) members. The diameters of the quasar pairs are as small as $5 h^{-1} \mathrm{Mpc}$ in the closest pairs; the number of close pairs of quasars is larger than the number of close random points. The lengths of the quasar systems are up to about 30-40 $h^{-1} \mathrm{Mpc}$; the highest values of diameters are up to $60 \mathrm{~h}^{-1} \mathrm{Mpc}$. In the whole diameter interval, the number of quasar systems with a given diameter is higher than that of random systems (except the highest values of diameters; the number of systems with diameters larger than $50 \mathrm{~h}^{-1} \mathrm{Mpc}$ is higher among random systems); the differences are the largest up to diameters $20 h^{-1} \mathrm{Mpc}$.

At larger linking lengths where $l \geq 40 h^{-1} \mathrm{Mpc}$ (we show this for $70 h^{-1} \mathrm{Mpc}$ ), the number of quasar systems with diameters up to $20 h^{-1} \mathrm{Mpc}$ is always larger than the number of random systems at these diameters. These systems are mostly pairs, but there are also richer systems among them. Starting from diameters $\approx 30 h^{-1} \mathrm{Mpc}$, the number of systems of different diameter in quasar and random catalogues becomes similar. 

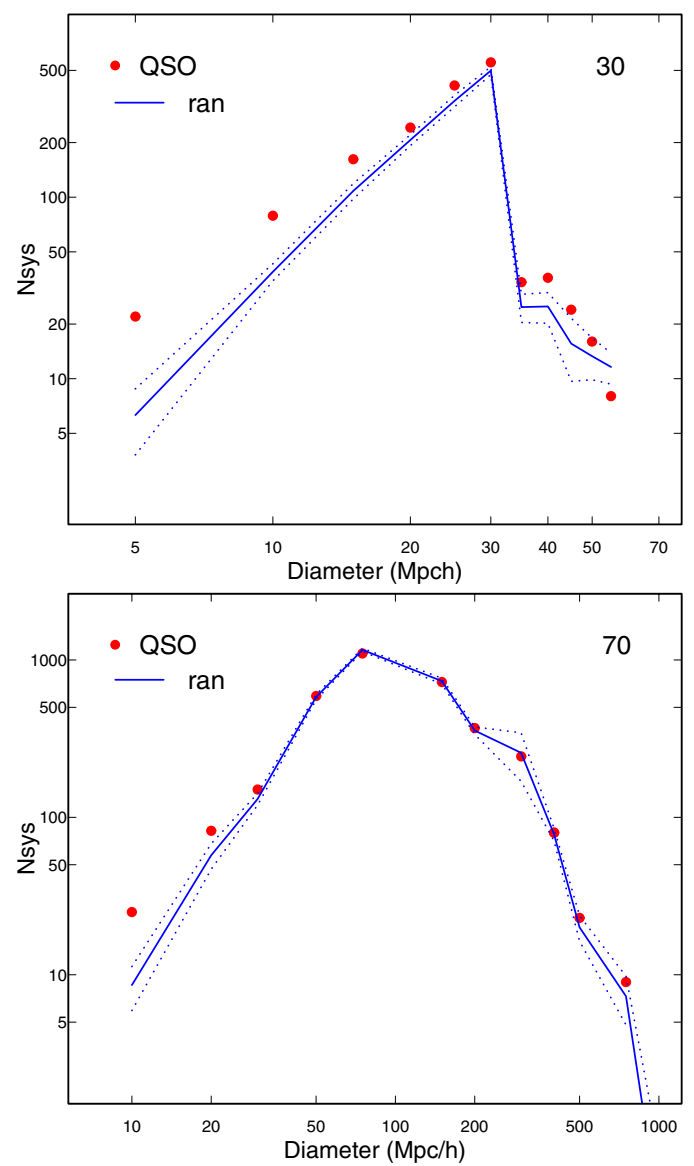

Fig. 3. Number of quasar (red dots) and random (blue lines) systems of different diameter for linking lengths 30 and $70 h^{-1} \mathrm{Mpc}$ (upper and lower panel, correspondingly).

The Kolmogorov-Smirnov (KS) test shows that the differences of the distributions of system sizes at the linking lengths 20 and $30 h^{-1} \mathrm{Mpc}$ between quasar and random samples are statistically significant $\left(p_{\mathrm{KS}}<0.003\right)$. At higher values of the linking lengths, the sizes of quasar systems with at least three members are statistically similar to those found in random samples. The diameters of quasar pairs at all linking lengths are smaller than diameters of pairs from random catalogues, and these differences are statistically significant $\left(p_{\mathrm{KS}}<0.05\right)$.

Therefore, the FoF analysis reveals that we obtain catalogues of real quasar systems in which the number of systems and system diameters differ from those found in random catalogues at linking lengths $l \leq 30$. There are relatively more close pairs among quasars than in random catalogues, which also causes a stronger correlation of quasars than random catalogues at small scales (Shen et al. 2007; Mountrichas et al. 2009; Donoso et al. 2010; Shen et al. 2013).

\subsection{Properties of quasar systems}

In Fig. 4 we show the median, and minimum and maximum values of quasar system diameters versus their richness at the linking lengths 50 and $70 \mathrm{~h}^{-1} \mathrm{Mpc}$. At the linking lengths 20 and $30 h^{-1} \mathrm{Mpc}$, we give the sizes of the largest systems in Tables A.1 and A.2.

At the linking lengths 20 and $30 \mathrm{~h}^{-1} \mathrm{Mpc}$, the sizes of quasar systems up to $30-60 h^{-1} \mathrm{Mpc}$ are comparable with the sizes of poor and medium rich galaxy superclusters in the local universe
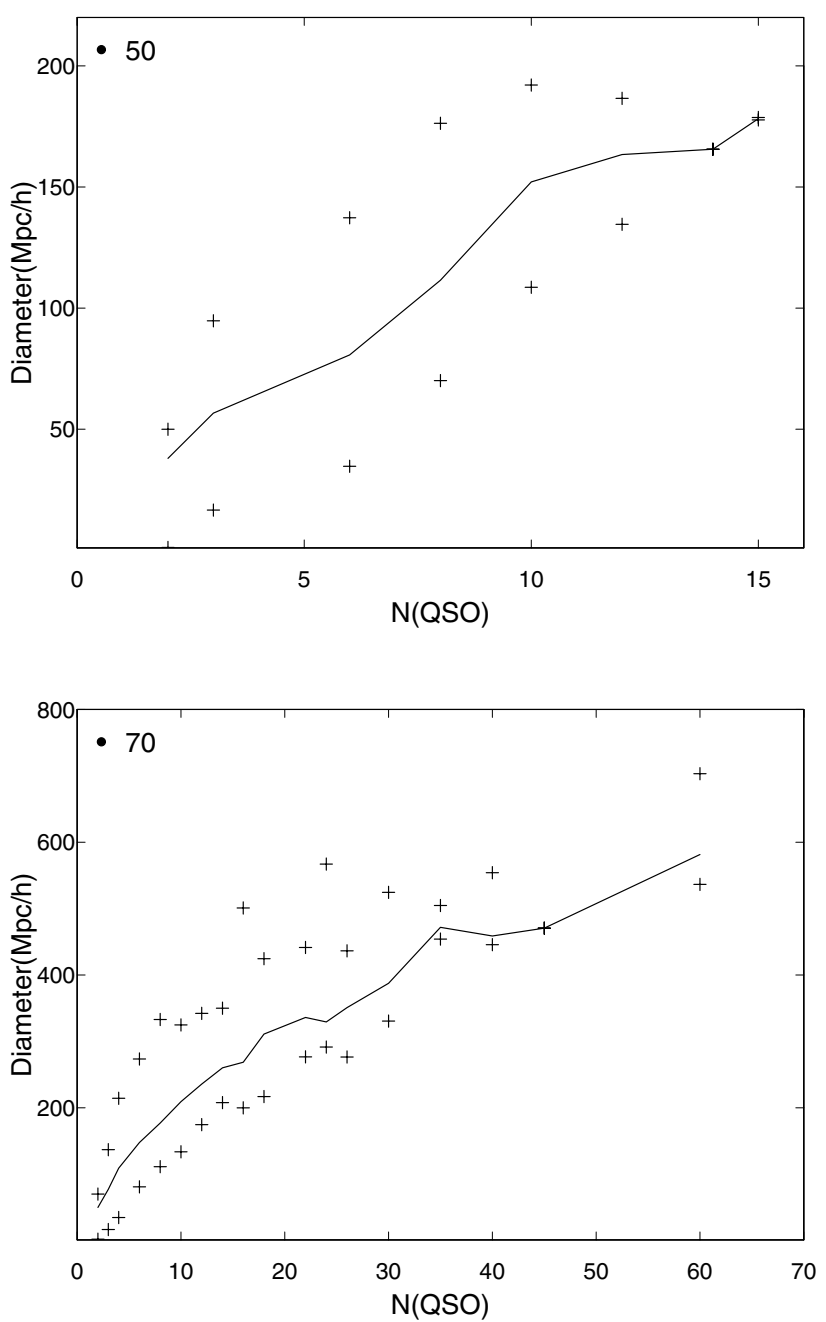

Fig. 4. System richness $N_{\mathrm{QSO}}$ vs. their diameter $D_{\max }$ for quasars for linking lengths 50 and $70 \mathrm{~h}^{-1} \mathrm{Mpc}$. Lines show median values of diameters; crosses denote the smallest and the largest diameters.

(Einasto et al. 1994, 1997; Jaaniste et al. 1998) with the number of Abell clusters $N_{\mathrm{cl}}<8$. At the linking lengths 40 and $50 \mathrm{~h}^{-1} \mathrm{Mpc}$, the richest quasar systems have up to 15 members. The sizes of the richest quasar systems, $\approx 200 h^{-1} \mathrm{Mpc}$, are comparable to the sizes of the richest superclusters in the local universe which typically contain 15-20 rich (Abell) galaxy clusters (Jaaniste et al. 1998; Einasto et al. 1994; Costa-Duarte et al. 2011; Schirmer et al. 2011; Liivamägi et al. 2012; Chon et al. 2013). The mean space density of quasar systems at these linking lengths is of order of $10^{-7}\left(h^{-1} \mathrm{Mpc}\right)^{-3}$, which is close to the mean space density of rich superclusters of Abell clusters with at least 10 member clusters (Einasto et al. 1997). The similarity of the mean space density of quasar systems and local rich superclusters was already noted by Komberg et al. (1996).

At the linking length $l=70 h^{-1} \mathrm{Mpc}$, rich quasar systems with at least 30 member quasars and a size at least of $300 h^{-1}$ Mpc (22 systems, Table A.3) are comparable in size with those described in Park et al. (2012) and in Luparello et al. (2011) as the richest systems in the local universe. The sizes of the largest quasar systems at $l=70 h^{-1} \mathrm{Mpc}, 500-700 h^{-1} \mathrm{Mpc}$ are comparable with the sizes of supercluster complexes penetrating the whole SDSS survey volume (Einasto et al. 2011c,b; Liivamägi et al. 2012). Einasto et al. (2011c) showed that the richest local supercluster complex, the Sloan Great Wall, 

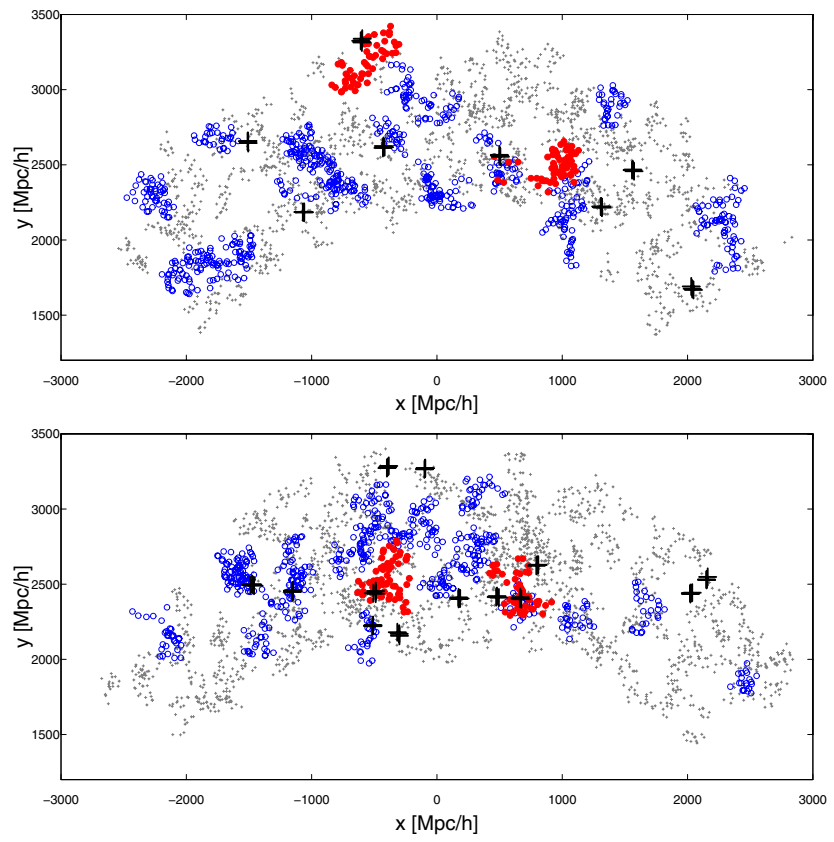

Fig. 5. Distribution of QSO systems at the linking length $70 h^{-1} \mathrm{Mpc}$ in $x$ and $y$ coordinates in two slices of $z$ coordinate (upper panel: $z \leq$ $0 h^{-1} \mathrm{Mpc}$, lower panel: $z>0 h^{-1} \mathrm{Mpc}$ ). Grey dots denote quasars in systems with $10 \leq N_{\mathrm{QSO}} \leq 24$, blue circles denote quasars in systems with $25 \leq N_{\mathrm{QSO}} \leq 49$, and red filled circles denote quasars in systems with $N_{\text {OSO }} \geq 50$. Black crosses denote quasar triplets at a linking length $20 h^{-1} \mathrm{Mpc}$.

consists of several rich superclusters. Individual superclusters in the Sloan Great Wall have different morphology and galaxy content; therefore, they form an assembly of physically different systems.

We also analysed luminosities of quasars in systems of different richness and found that the luminosities of quasars in systems on average do not depend on system richness. The luminosities of quasars in systems, and those of single quasars are similar.

\subsection{Individual quasar systems}

In Fig. 5 we show the distribution of quasars in systems of various richness at the linking length $70 \mathrm{~h}^{-1} \mathrm{Mpc}$ in Cartesian coordinates $x, y$, and $z$ as defined in Park et al. (2007) and in Liivamägi et al. (2012):

$$
\begin{aligned}
& x=-d \sin \lambda, \\
& y=d \cos \lambda \cos \eta, \\
& z=d \cos \lambda \sin \eta,
\end{aligned}
$$

where $d$ is the comoving distance, and $\lambda$ and $\eta$ are the SDSS survey coordinates. We also plot quasars from the richest systems at $l=20 h^{-1} \mathrm{Mpc}$ (quasar triplets) in the figure. The richest quasar systems at $l=20 h^{-1} \mathrm{Mpc}$ are almost all relatively isolated and do not belong to very rich quasar systems at higher linking lengths. This can be seen in Table A.1 where we give the richness of systems to which they belong at $l=70 h^{-1} \mathrm{Mpc}$. Table A.1 shows that triple quasar systems are located in two relatively narrow redshift intervals at $z \approx 1.2$, and at $z \approx 1.7$.

In Fig. 5 and Table A.3, four systems at the linking length $l=70 h^{-1} \mathrm{Mpc}$ consist of more than 50 quasars and have diameters larger than $500 \mathrm{~h}^{-1} \mathrm{Mpc}$, which exceeds the size of
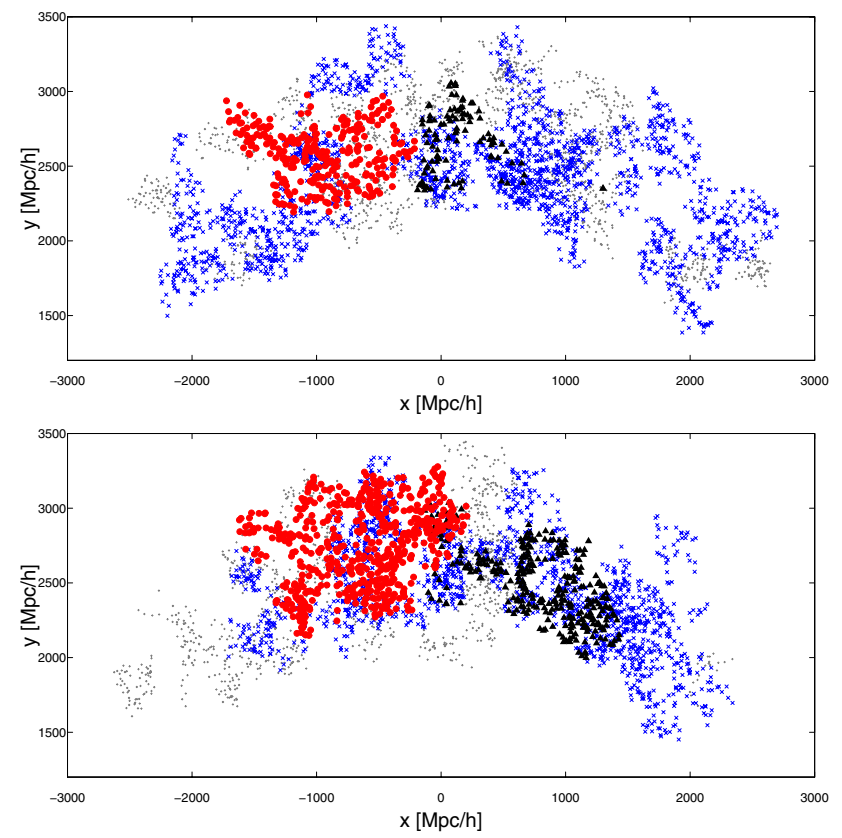

Fig. 6. Distribution of QSO systems at the linking length $80 \mathrm{~h}^{-1} \mathrm{Mpc}$ in $x$ and $y$ coordinates in two slices of the $z$ coordinate (upper panel: $z \leq 0 h^{-1} \mathrm{Mpc}$, lower panel: $z>0 h^{-1} \mathrm{Mpc}$ ). Grey dots denote quasars in systems with $50 \leq N_{\mathrm{QSO}}<100$; blue circles denote quasars in systems with $100 \leq N_{\mathrm{QSO}} \leq 400$, black triangles denote quasars in the second richest system with $N_{\mathrm{QSO}}=421$, and red filled circles denote quasars in the richest system with $N_{\text {QSO }}=920$.

the richest local supercluster complex, the Sloan Great Wall. The richest system at the linking length $l=70 h^{-1} \mathrm{Mpc}$ at $x \approx 1000 h^{-1} \mathrm{Mpc}$ and $y \approx 2500 h^{-1} \mathrm{Mpc}$ is the Huge-LQG described in Clowes et al. (2013). In our catalogue this system has 67 member quasars, and it is $\approx 700 h^{-1} \mathrm{Mpc}$ in diameter. Below this system at $y \approx 2000 h^{-1} \mathrm{Mpc}$, there are two quasar groups, which coincide with those from Clowes et al. (2012).

Figure 6 shows the distribution of quasar systems at the linking length $80 h^{-1} \mathrm{Mpc}$. The members of the two largest systems are shown. This figure shows that the richest system form at $x<0 h^{-1} \mathrm{Mpc}$. This is the area, where the space density of quasars at large scales is the highest. At this linking length, the richest quasar systems found at $l=70 h^{-1} \mathrm{Mpc}$ is a member of the second richest system.

\subsection{Large-scale distribution of quasar systems}

Visual inspection of Fig. 5 shows that there are underdense regions between rich quasar systems with diameters of about $400 h^{-1} \mathrm{Mpc}$ in some areas of the figure (e.g. in the upper panel between $-1000<x<1000 h^{-1} \mathrm{Mpc}$ ). The size of underdense regions in this figure is much larger than the sizes of typical large voids in the local Universe (Einasto et al. 2011a, and references therein) but is close to the sizes of the largest voids covered by SDSS survey (Einasto et al. 2011b; Park et al. 2012).

Miller et al. (2004) calculated the density field of quasars from $2 \mathrm{dF}$ QSO redshift survey and showed the presence of density fluctuations at scales of about $200 \mathrm{~h}^{-1} \mathrm{Mpc}$ by visual inspection of the density field plots. We shall analyse the large scale distribution of quasar systems in detail in another study. 


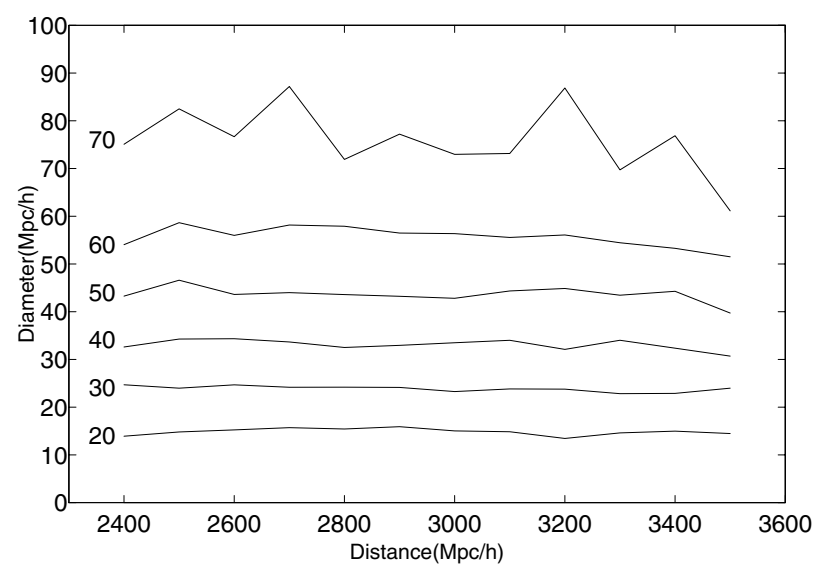

Fig. 7. Median diameters of quasar systems at linking lengths 20$70 \mathrm{~h}^{-1} \mathrm{Mpc}$ vs. the distance of systems.

\section{Discussion and conclusions}

\subsection{Selection effects}

We analysed the diameters of quasar and random systems at different distances to see whether the properties of quasar systems found with the FoF method are affected by distance-dependent selection effects. Figure 7 shows the median diameters of quasar systems at the linking lengths $20-70 h^{-1} \mathrm{Mpc}$ versus their distances. The median diameters of quasar systems are not correlated with distance. We also confirmed this by correlation tests. We obtained similar results with systems from random catalogues. Apparent decrease of the median diameters of quasar systems at the linking length $70 h^{-1} \mathrm{Mpc}$ at high distances is due to an edge effect: close to the sample boundaries FoF cannot find very large systems near sample edges. This edge effect is much stronger than the influence of the slight density decrease to the systems properties. Due to the edge effect, we cannot search for quasar systems dividing the volume under study into narrow slices; this might minimise other selection effects but makes the edge effects very strong. The edge effect affects quasar and random systems in the same way and may decrease the number of the largest quasar and random systems at large linking lengths. At the linking length $70 \mathrm{~h}^{-1} \mathrm{Mpc}$, wiggles in the distribution of the system diameters are due to the largest quasar systems. These wiggles were also seen in Fig. 1.

Therefore the slight decrease of the number density of quasars with distance do not significantly affect the properties of quasar systems. It is possible that two effects cancel each other out. The possible increase of quasar systems due to the decrease of the density, and the decrease of systems due to cosmic evolution of systems at high redshift (Suhhonenko et al. 2011). We plan to study quasar and web evolution from simulations to understand better the evolutionary effects.

With SDSS Visual Tools, we checked the images of selected quasars. We looked at images of all quasars in systems with more than two members at the linking lengths $l=20$ and $30 h^{-1} \mathrm{Mpc}$ and in the richest systems at the linking length $70 h^{-1} \mathrm{Mpc}$. We did not detect contaminated images in these data.

\subsection{Discussion}

The number of quasar systems, their sizes, and richness at the linking lengths $l \geq 40 h^{-1} \mathrm{Mpc}$ are comparable with the sizes and richness of random systems. Therefore, their presence do not violate homogeneity of the universe at very large scales,

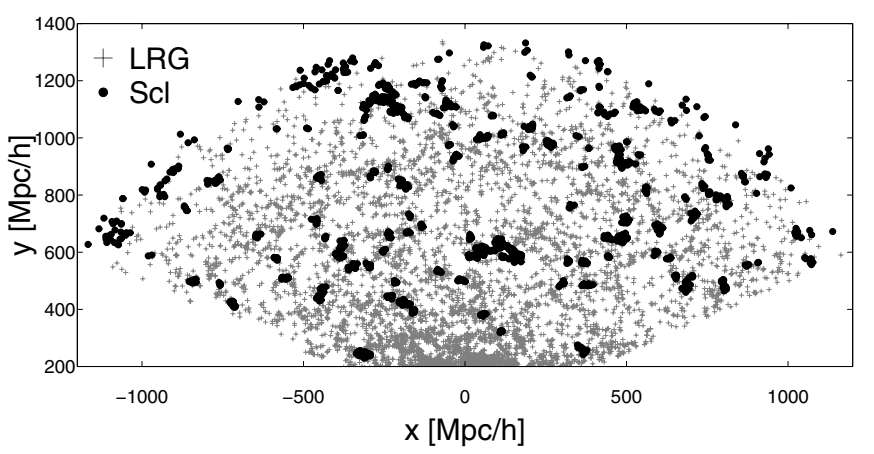

Fig. 8. Distribution of luminous red galaxies (LRG, light symbols) and their superclusters ( $\mathrm{Scl}$, dark symbols) in the $x, y$ coordinates in a slice of the $z$-coordinate $-20 \leq z \leq 20 h^{-1}$ Mpc.

as claimed by Clowes et al. (2013). Similar conclusions were reached by Nadathur (2013). The transition scale to homogeneity has been discussed in many studies (see Einasto \& Gramann 1993; Martinez et al. 1998; Clowes et al. 2013, for details and references). Clowes et al. (2013) also mentioned that the richest quasar systems - and therefore, the highest large-scale density regions in the Universe - are located in the area of the HugeLQG. In our study, we found several quasar systems comparable in richness with the Huge-LQG in other regions of space, where the percolating systems also appear. Therefore, the Huge-LQG and other LQGs near it form one of several complexes of rich quasar systems and not the richest one.

At the linking length $l=70 h^{-1} \mathrm{Mpc}$, the sizes of the largest quasar systems are comparable with the sizes of supercluster complexes that penetrate the whole SDSS survey volume (Liivamägi et al. 2012). To illustrate this, we plot the distribution of luminous red galaxies and their superclusters from SDSS in $x$, $y$ coordinates in Fig. 8 (Liivamägi et al. 2012). An eye can connect the centres of superclusters into huge systems across the whole volume under study, which are analogues to the largest quasar systems.

The mean space density of quasars is approximately $1.1 \times$ $10^{-6}\left(h^{-1} \mathrm{Mpc}\right)^{-3}$. This is much lower than, for example, the mean space density of nearby galaxy groups in a volume limited sample with a luminosity limit of $M_{r}<=-21.0,2 \times$ $10^{-4}\left(h^{-1} \mathrm{Mpc}\right)^{-3}$ (Tempel et al. 2014). An analysis of the density enhancements around quasars have shown that quasars can be found in overdense environment, which corresponds to small groups or poor (Abell class 0) clusters of galaxies with masses $\approx 10^{12} M_{\text {sun }} h^{-1}$ (Wold et al. 2000; Trainor \& Steidel 2012; Shen et al. 2013; Karhunen et al. 2014; Morselli et al. 2014). The small space density of quasars and the mass limit of quasar host haloes tell us that quasars are rare events in small groups rather than common events in rare, very rich clusters (Trainor \& Steidel 2012, 2013). A certain fraction of the group-sized haloes undergo a short (about $10^{7}$ years, DiPompeo et al. 2014) quasar phase. Different physical processes, which are still not well understood, can trigger a mass flow onto the central super-massive black hole (Portinari et al. 2012; Krumpe et al. 2013).

Simulations have shown that the most luminous quasars do not reside in the most massive dark matter haloes at any redshift (Fanidakis et al. 2013). Quasars host haloes with masses that are typically about $10^{12} M_{\text {sun }} h^{-1}$. It seems to be quite constant as a function of redshift (see also Shen et al. 2013, and references therein). The reason for this is assumed to be active galaxy nucleus (AGN) feedback mechanism in haloes with $M>$ $10^{12} M_{\text {sun }} h^{-1}$ (Fanidakis et al. 2013, and references therein). 
Simulations indicate that at $z=6$ the very first quasar haloes with a mass of $10^{12} M_{\text {sun }} h^{-1}$ are just forming (Heinämäki et al. 2005; Fanidakis et al. 2013). The majority of the descendants of $z=6$ quasars are located in the haloes, which evolve into rich galaxy clusters in the local Universe (Angulo et al. 2012; Fanidakis et al. 2013). At lower redshifts, typical quasar host haloes correspond to groups of galaxies.

In the local universe, quasars lie in the outskirts of galaxy superclusters (Lietzen et al. 2009, 2011). In a low-density environment, a higher fraction of galaxies hosts AGNs than in highdensity environment (Kauffmann et al. 2004). The clustering of high-redshift quasars is stronger than the clustering of their low redshift counterparts (Shen et al. 2007).

There are only a few galaxy superclusters known at high redshifts (Nakata et al. 2005; Swinbank et al. 2007; Lubin et al. 2009; Lee et al. 2014). The study of the supercluster $\mathrm{Cl} 1604$ at $z=0.9$ (Gilbank et al. 2008) showed that AGNs in this supercluster are located in intermediate density environments, in the outskirts of massive clusters or within poorer clusters and groups, and they avoid the densest environments (the richest clusters) in the supercluster (Kocevski et al. 2009a,b). The quasar from our sample, at RA $=240.27$ degrees, Dec $=$ 42.74 degrees, and redshift $z=1.05$ may be located in the outskirts of this supercluster.

The quasar systems found in our study may mark the location of galaxy superclusters at high redshifts. The quasars are typically not located in the richest galaxy clusters (in the highest density environments) in superclusters. The mean space density of quasar systems at small linking lengths is of order of $10^{-7}\left(h^{-1} \mathrm{Mpc}\right)^{-3}$, which is close to the mean space density of rich superclusters of Abell clusters (Einasto et al. 1997) in support of this suggestion.

\subsection{Summary and conclusions}

We summarise our results as follows:

1) Quasar systems differ from systems found in a random distribution at the linking lengths $l \leq 30 \mathrm{~h}^{-1} \mathrm{Mpc}$; their diameters (up to 30-60 $h^{-1} \mathrm{Mpc}$ ) are comparable to the diameters of poor galaxy superclusters in the local Universe. The richest systems have four member quasars. The mean space density of quasar systems, $\approx 10^{-7}\left(h^{-1} \mathrm{Mpc}\right)^{-3}$, is close to the mean space density of local rich superclusters.

2) In a linking length interval, $40 \leq l \leq 70 h^{-1} \mathrm{Mpc}$, the richness and diameters of the richest quasar systems are comparable to those found in random catalogues. The diameters of richest quasar systems $\left(\approx 200-700 h^{-1} \mathrm{Mpc}\right)$ are comparable to the diameters of rich galaxy superclusters and supercluster chains in the local Universe.

3) Quasar systems begin to join into percolating system at the linking lengths $l \geq 80 \mathrm{~h}^{-1} \mathrm{Mpc}$, while the percolation occurs at a larger linking length in random catalogues.

4) At the linking length $l=70 h^{-1} \mathrm{Mpc}$ three systems from our catalogue coincide with the large quasar groups from Clowes et al. (2012, 2013). At this linking length the largest systems with diameters exceeding $500 \mathrm{~h}^{-1} \mathrm{Mpc}$ are comparable to the supercluster complexes in the local Universe, which penetrate the whole sample volume.

Quasar system catalogues serve as a database for searching distant superclusters of galaxies and for studying the cosmic web at high redshifts.

Deeper and wider galaxy surveys are needed to determine a quasar's role in the evolution of the large-scale structure of the
Universe. We plan to continue our study of quasars and their systems by using observational data and simulations to better understand how quasars trace the cosmic web.

Acknowledgements. We thank our referee for comments and suggestions that helped to improve the paper. We are pleased to thank the SDSS Team for the publicly available data releases. Funding for the Sloan Digital Sky Survey (SDSS) and SDSS-II has been provided by the Alfred P. Sloan Foundation, the Participating Institutions, the National Science Foundation, the US Department of Energy, the National Aeronautics and Space Administration, the Japanese Monbukagakusho, and the Max Planck Society, and the Higher Education Funding Council for England. The SDSS Web site is http://www.sdss . org/. The SDSS is managed by the Astrophysical Research Consortium (ARC) for the Participating Institutions. The Participating Institutions are the American Museum of Natural History, Astrophysical Institute Potsdam, University of Basel, University of Cambridge, Case Western Reserve University, The University of Chicago, Drexel University, Fermilab, the Institute for Advanced Study, the Japan Participation Group, The Johns Hopkins University, the Joint Institute for Nuclear Astrophysics, the Kavli Institute for Particle Astrophysics and Cosmology, the Korean Scientist Group, the Chinese Academy of Sciences (LAMOST), Los Alamos National Laboratory, the Max-PlanckInstitute for Astronomy (MPIA), the Max-Planck-Institute for Astrophysics (MPA), New Mexico State University, Ohio State University, University of Pittsburgh, University of Portsmouth, Princeton University, the United States Naval Observatory, and the University of Washington. The present study was supported by the Estonian Ministry for Education and Science research project SF0060067s08, by ETAG project IUT26-2, and by the European Structural Funds grant for the Centre of Excellence "Dark Matter in (Astro)particle Physics and Cosmology" TK120. H. Lietzen acknowledges financial support from the Spanish Ministry of Economy and Competitiveness (MINECO) under the 2011 Severo Ochoa Program MINECO SEV-2011-0187. This work has also been supported by ICRAnet through a professorship for Jaan Einasto. C. Park and H. Song thank Korea Institute for Advanced Study for providing computing resources (KIAS Center for Advanced Computation Linux Cluster System).

\section{References}

Angulo, R. E., Springel, V., White, S. D. M., et al. 2012, MNRAS, 425, 2722 Chon, G., Böhringer, H., \& Nowak, N. 2013, MNRAS, 429, 3272

Clowes, R. G., \& Campusano, L. E. 1991, MNRAS, 249, 218

Clowes, R. G., Campusano, L. E., Graham, M. J., \& Söchting, I. K. 2012, MNRAS, 419, 556

Clowes, R. G., Harris, K. A., Raghunathan, S., et al. 2013, MNRAS, 429, 2910

Coldwell, G. V., \& Lambas, D. G. 2006, MNRAS, 371, 786

Costa-Duarte, M. V., Sodré, Jr., L., \& Durret, F. 2011, MNRAS, 411, 1716

DiPompeo, M. A., Myers, A. D., Hickox, R. C., Geach, J. E., \& Hainline, K. N. 2014, MNRAS, 442, 3443

Donoso, E., Li, C., Kauffmann, G., Best, P. N., \& Heckman, T. M. 2010, MNRAS, 407, 1078

Dressler, A. 1980, ApJ, 236, 351

Einasto, M., \& Einasto, J. 1987, MNRAS, 226, 543

Einasto, J., \& Gramann, M. 1993, ApJ, 407, 443

Einasto, J., Saar, E., Kaasik, A., \& Chernin, A. D. 1974, Nature, 252, 111

Einasto, M., Einasto, J., Tago, E., Dalton, G. B., \& Andernach, H. 1994, MNRAS, 269, 301

Einasto, M., Tago, E., Jaaniste, J., Einasto, J., \& Andernach, H. 1997, A\&AS, 123,119

Einasto, M., Einasto, J., Tago, E., Müller, V., \& Andernach, H. 2001, AJ, 122, 2222

Einasto, J., Suhhonenko, I., Hütsi, G., et al. 2011a, A\&A, 534, A128

Einasto, M., Liivamägi, L. J., Tago, E., et al. 2011b, A\&A, 532, A5

Einasto, M., Liivamägi, L. J., Tempel, E., et al. 2011c, ApJ, 736, 51

Einasto, M., Lietzen, H., Tempel, E., et al. 2014, A\&A, 562, A87

Eke, V. R., Baugh, C. M., Cole, S., et al. 2004, MNRAS, 348, 866

Falomo, R., Farina, E. P., Decarli, R., Treves, A., \& Kotilainen, J. 2013, in IAU Symp. 295, eds. D. Thomas, A. Pasquali, \& I. Ferreras, 56

Falomo, R., Bettoni, D., Karhunen, K., Kotilainen, J. K., \& Uslenghi, M. 2014, MNRAS, 440, 476

Fanidakis, N., Macciò, A. V., Baugh, C. M., Lacey, C. G., \& Frenk, C. S. 2013, MNRAS, 436, 315

Floyd, D. J. E., Dunlop, J. S., Kukula, M. J., et al. 2013, MNRAS, 429, 2

Gilbank, D. G., Yee, H. K. C., Ellingson, E., et al. 2008, ApJ, 677, L89

Heinämäki, P., Suhhonenko, I., Saar, E., et al. 2005, unpublished [arXiv:astro-ph/0507197]

Huchra, J. P., \& Geller, M. J. 1982, ApJ, 257, 423

Hutchings, J. B., Frenette, D., Hanisch, R., et al. 2002, AJ, 123, 2936

Hutchings, J. B., Scholz, P., \& Bianchi, L. 2009, AJ, 137, 3533 
Jaaniste, J., Tago, E., Einasto, M., et al. 1998, A\&A, 336, 35

Karhunen, K., Kotilainen, J. K., Falomo, R., \& Bettoni, D. 2014, MNRAS, 441, 1802

Kauffmann, G., White, S. D. M., Heckman, T. M., et al. 2004, MNRAS, 353, 713

Klypin, A., \& Shandarin, S. F. 1993, ApJ, 413, 48

Kocevski, D. D., Lubin, L. M., Gal, R., et al. 2009a, ApJ, 690, 295

Kocevski, D. D., Lubin, L. M., Lemaux, B. C., et al. 2009b, ApJ, 700, 901

Komberg, B. V., Kravtsov, A. V., \& Lukash, V. N. 1996, MNRAS, 282, 713

Kotilainen, J. K., Falomo, R., Decarli, R., et al. 2009, ApJ, 703, 1663

Kotilainen, J., Falomo, R., Bettoni, D., Karhunen, K., \& Uslenghi, M. 2013 [arXiv: 1302.1366]

Krumpe, M., Miyaji, T., \& Coil, A. L. 2013, Acta Polytechnica, submitted [arXiv: 1308.5976]

Lee, K.-S., Dey, A., Hong, S., et al. 2014, ApJ, submitted [arXiv: 1405.2620]

Letawe, Y., Letawe, G., \& Magain, P. 2010, MNRAS, 403, 2088

Lietzen, H., Heinämäki, P., Nurmi, P., et al. 2009, A\&A, 501, 145

Lietzen, H., Heinämäki, P., Nurmi, P., et al. 2011, A\&A, 535, A21

Lietzen, H., Tempel, E., Heinämäki, P., et al. 2012, A\&A, 545, A104

Liivamägi, L. J., Tempel, E., \& Saar, E. 2012, A\&A, 539, A80

Loeb, A. 2008 [arXiv:0804 .2258]

Lubin, L. M., Gal, R. R., Lemaux, B. C., Kocevski, D. D., \& Squires, G. K. 2009, AJ, 137, 4867

Luparello, H., Lares, M., Lambas, D. G., \& Padilla, N. 2011, MNRAS, 415, 964

Martinez, V. J., Pons-Borderia, M.-J., Moyeed, R. A., \& Graham, M. J. 1998, MNRAS, 298, 1212

Miller, L., Croom, S. M., Boyle, B. J., et al. 2004, MNRAS, 355, 385

Mo, H. J., Einasto, M., Xia, X. Y., \& Deng, Z. G. 1992, MNRAS, 255, 382

Morselli, L., Mignoli, M., Gilli, R., et al. 2014, A\&A, 568, A1

Mountrichas, G., Sawangwit, U., Shanks, T., et al. 2009, MNRAS, 394, 2050

Nadathur, S. 2013, MNRAS, 434, 398

Nakata, F., Kodama, T., Shimasaku, K., et al. 2005, MNRAS, 357, 1357

Padmanabhan, N., White, M., Norberg, P., \& Porciani, C. 2009, MNRAS, 397, 1862
Park, C., \& Choi, Y.-Y. 2009, ApJ, 691, 1828

Park, C., Choi, Y., Vogeley, M. S., Gott, III, J. R., \& Blanton, M. R. 2007, ApJ, 658,898

Park, C., Choi, Y.-Y., Kim, J., et al. 2012, ApJ, 759, L7

Portinari, L., Kotilainen, J., Falomo, R., \& Decarli, R. 2012, MNRAS, 420, 732

Richards, G. T., Strauss, M. A., Fan, X., et al. 2006, AJ, 131, 2766

Schirmer, M., Hildebrandt, H., Kuijken, K., \& Erben, T. 2011, A\&A, 532, A57

Schneider, D. P., Richards, G. T., Hall, P. B., et al. 2010, AJ, 139, 2360

Shen, Y., Strauss, M. A., Oguri, M., et al. 2007, AJ, 133, 2222

Shen, Y., McBride, C. K., White, M., et al. 2013, ApJ, 778, 98

Skibba, R. A. 2009, MNRAS, 392, 1467

Söchting, I. K., Clowes, R. G., \& Campusano, L. E. 2004, MNRAS, 347, 1241

Soechting, I. K., Clowes, R. G., \& Campusano, L. E. 2002, BAAS, 34, 649

Suhhonenko, I., Einasto, J., Liivamägi, L. J., et al. 2011, A\&A, 531, A149

Swinbank, A. M., Edge, A. C., Smail, I., et al. 2007, MNRAS, 379, 1343

Tago, E., Einasto, J., Saar, E., et al. 2006, Astron. Nachr., 327, 365

Tago, E., Einasto, J., Saar, E., et al. 2008, A\&A, 479, 927

Tempel, E., Saar, E., Liivamägi, L. J., et al. 2011, A\&A, 529, A53

Tempel, E., Tago, E., \& Liivamägi, L. J. 2012, A\&A, 540, A106

Tempel, E., Tamm, A., Gramann, M., et al. 2014, A\&A, 566, A1

Trainor, R. F., \& Steidel, C. C. 2012, ApJ, 752, 39

Trainor, R., \& Steidel, C. C. 2013, ApJ, 775, L3

Tucker, D. L., Oemler, Jr., A., Hashimoto, Y., et al. 2000, ApJS, 130, 237

Ueda, S., Hayashida, K., Anabuki, N., et al. 2013, ApJ, 778, 33

Vanden Berk, D. E., Schneider, D. P., Richards, G. T., et al. 2005, AJ, 129, 2047

van de Weygaert, R., \& Schaap, W. 2009, in Data Analysis in Cosmology, eds. V. J. Martínez, E. Saar, E. Martínez-González, \& M.-J. Pons-Bordería (Berlin: Springer Verlag), Lect. Notes Phys., 665, 291

Webster, A. 1982, MNRAS, 199, 683

Williger, G. M., Campusano, L. E., Clowes, R. G., \& Graham, M. J. 2002, ApJ, 578,708

Wold, M., Lacy, M., Lilje, P. B., \& Serjeant, S. 2000, MNRAS, 316, 267

Zeldovich, I. B., Einasto, J., \& Shandarin, S. F. 1982, Nature, 300, 407 


\section{Appendix A: Data on quasar systems}

Table A.1. Data on QSO systems with $N_{\mathrm{QSO}}=3$ at linking length $20 h^{-1} \mathrm{Mpc}$.

\begin{tabular}{rrrrrrrr}
\hline \hline$(1)$ & $\begin{array}{r}(2) \\
\text { RA }\end{array}$ & $\begin{array}{r}(3) \\
\text { Dec } \\
{[\mathrm{deg}]}\end{array}$ & $\begin{array}{r}(4) \\
\lambda\end{array}$ & $\begin{array}{r}(5) \\
\eta \mathrm{deg}]\end{array}$ & $\begin{array}{r}(6) \\
{[\mathrm{deg}]}\end{array}$ & $\begin{array}{r}(7) \\
\text { Diameter } \\
{\left[h^{-1} \mathrm{Mpc}\right]}\end{array}$ & $\begin{array}{r}(8) \\
N_{70}\end{array}$ \\
\hline 22 & 127.99 & 45.66 & -35.88 & 29.48 & 1.76 & 29.9 & 4 \\
36 & 132.41 & 38.17 & -38.63 & 19.79 & 1.75 & 22.0 & 10 \\
53 & 135.41 & 8.50 & -48.85 & -19.51 & 1.21 & 31.0 & 5 \\
124 & 149.80 & 22.13 & -32.27 & -6.04 & 1.36 & 17.6 & 9 \\
161 & 157.01 & 5.17 & -27.86 & -26.63 & 1.28 & 16.6 & 3 \\
166 & 158.73 & 52.24 & -15.71 & 22.72 & 1.38 & 17.6 & 3 \\
167 & 159.08 & 56.72 & -13.87 & 26.94 & 1.26 & 22.7 & 5 \\
215 & 166.34 & 56.22 & -10.24 & 25.13 & 1.21 & 21.7 & 3 \\
251 & 173.48 & 19.62 & -10.83 & -12.49 & 1.19 & 14.9 & 5 \\
272 & 177.85 & 57.90 & -3.78 & 25.61 & 1.19 & 19.0 & 9 \\
318 & 187.09 & 36.84 & 1.67 & 4.36 & 1.62 & 22.5 & 17 \\
350 & 193.55 & 37.31 & 6.79 & 5.12 & 1.64 & 20.9 & 3 \\
354 & 194.57 & 19.71 & 9.01 & -12.53 & 1.21 & 18.1 & 3 \\
366 & 196.17 & 24.45 & 10.15 & -7.63 & 1.72 & 27.8 & 5 \\
379 & 198.68 & 58.00 & 7.20 & 26.23 & 1.04 & 29.2 & 8 \\
415 & 205.47 & 60.14 & 10.02 & 29.23 & 1.30 & 24.2 & 9 \\
430 & 207.98 & 2.79 & 22.95 & -29.46 & 1.22 & 11.0 & 4 \\
435 & 209.40 & 62.39 & 11.03 & 32.03 & 1.19 & 11.1 & 28 \\
456 & 213.77 & 11.32 & 28.16 & -19.62 & 1.55 & 13.8 & 21 \\
472 & 216.68 & 36.76 & 24.88 & 8.77 & 1.23 & 24.9 & 6 \\
544 & 235.02 & 54.57 & 26.37 & 32.93 & 1.65 & 29.9 & 14 \\
\hline
\end{tabular}

Notes. Columns are as follows: (1) ID of the system; (2-3) system right ascension and declination; (4-5) system SDSS coordinates $\lambda$ and $\eta$; (6) mean redshift of the system; (7) system size; (8) the richness of a systems to which a given system belongs at the linking length $70 h^{-1}$ Mpc.

Table A.2. Data on QSO systems with $N_{\mathrm{QSO}}=4$ at linking length $30 h^{-1} \mathrm{Mpc}$.

\begin{tabular}{rrrrrrrr}
\hline \hline$(1)$ & $\begin{array}{r}(2) \\
\text { RA }\end{array}$ & $\begin{array}{r}(3) \\
\text { Dec } \\
\text { ID }\end{array}$ & $\begin{array}{r}(4) \\
\lambda\end{array}$ & $\begin{array}{r}(5) \\
\eta \mathrm{deg}]\end{array}$ & $\begin{array}{r}(6) \\
{[\mathrm{deg}]}\end{array}$ & $\begin{array}{r}(7) \\
\text { Diameter } \\
{\left[h^{-1} \mathrm{Mpc}\right]}\end{array}$ & $\begin{array}{r}(8) \\
N_{70}\end{array}$ \\
\hline 50 & 128.06 & 45.73 & -35.79 & 29.50 & 1.77 & 34.97 & 4 \\
122 & 135.29 & 8.64 & -48.94 & -19.27 & 1.21 & 44.55 & 5 \\
158 & 137.94 & 8.32 & -46.41 & -20.37 & 1.53 & 43.11 & 30 \\
195 & 140.69 & 14.19 & -42.62 & -13.02 & 1.46 & 49.47 & 28 \\
496 & 162.75 & 57.62 & -11.69 & 27.09 & 1.10 & 36.37 & 8 \\
501 & 162.57 & 15.90 & -21.52 & -15.36 & 1.25 & 38.32 & 67 \\
657 & 173.32 & 19.64 & -10.98 & -12.47 & 1.18 & 37.74 & 5 \\
721 & 178.13 & 57.89 & -3.64 & 25.57 & 1.19 & 37.44 & 9 \\
1135 & 208.12 & 2.89 & 23.09 & -29.34 & 1.22 & 34.70 & 4 \\
1182 & 212.85 & 58.62 & 14.07 & 29.17 & 1.26 & 58.89 & 16 \\
1477 & 238.18 & 48.25 & 32.21 & 29.36 & 1.74 & 44.47 & 5 \\
1490 & 239.04 & 7.08 & 53.44 & -20.55 & 1.10 & 34.74 & 4 \\
\hline
\end{tabular}

Notes. Columns are the same as in Table A.1. 
A\&A 568, A46 (2014)

Table A.3. Data on QSO systems with $N_{\mathrm{QSO}} \geq 30$ at linking length $70 h^{-1} \mathrm{Mpc}$.

\begin{tabular}{rrrrrrrrr}
\hline \hline$(1)$ & $(2)$ & $\begin{array}{r}(3) \\
\text { RA }\end{array}$ & $\begin{array}{r}(4) \\
\text { Dec } \\
{[\mathrm{deg}]}\end{array}$ & $\begin{array}{r}(5) \\
\lambda\end{array}$ & $\begin{array}{r}(6) \\
{[\mathrm{deg}]}\end{array}$ & $\begin{array}{r}(7) \\
{[\mathrm{deg}]}\end{array}$ & $\begin{array}{r}(8) \\
\text { Diameter } \\
{\left[h^{-1} \mathrm{Mpc}\right]}\end{array}$ & $\begin{array}{r}(9) \\
\text { Comments }\end{array}$ \\
\hline 356 & 30 & 138.06 & 7.86 & -46.34 & -21.09 & 1.55 & 346.8 & \\
1044 & 33 & 157.76 & 20.72 & -25.33 & -9.44 & 1.59 & 336.5 & \\
1093 & 34 & 161.31 & 3.86 & -23.61 & -28.28 & 1.11 & 403.5 & $\mathrm{C} 3$ \\
1156 & 56 & 166.95 & 33.83 & -14.86 & 2.74 & 1.11 & 548.3 & \\
1159 & 31 & 162.14 & 5.47 & -22.73 & -26.55 & 1.28 & 389.1 & $\mathrm{C} 2$ \\
1195 & 67 & 163.63 & 14.56 & -20.59 & -16.89 & 1.27 & 703.1 & $\mathrm{C} 1$ \\
1593 & 31 & 176.48 & 38.26 & -6.66 & 6.09 & 1.28 & 330.5 & \\
1598 & 32 & 177.43 & 32.67 & -6.38 & 0.41 & 1.18 & 457.3 & \\
1771 & 33 & 183.60 & 11.03 & -1.35 & -21.44 & 1.08 & 524.5 & \\
2002 & 36 & 189.03 & 44.08 & 2.86 & 11.68 & 1.39 & 504.6 & \\
2011 & 37 & 189.46 & 20.28 & 4.18 & -12.14 & 1.46 & 454.0 & \\
2190 & 55 & 196.49 & 27.06 & 10.23 & -4.93 & 1.59 & 614.6 & \\
2203 & 64 & 196.42 & 39.90 & 8.78 & 8.00 & 1.14 & 536.4 & \\
2287 & 31 & 199.49 & 54.73 & 8.30 & 23.16 & 1.48 & 381.9 & \\
2440 & 41 & 205.31 & 50.40 & 12.65 & 19.76 & 1.39 & 554.1 & \\
2454 & 31 & 202.67 & 21.32 & 16.41 & -10.20 & 1.08 & 375.2 & \\
2961 & 30 & 217.23 & 10.45 & 31.63 & -20.20 & 1.68 & 387.5 & \\
3205 & 38 & 227.57 & 41.44 & 30.42 & 17.69 & 1.54 & 471.7 & \\
3219 & 46 & 226.66 & 16.73 & 39.53 & -10.59 & 1.09 & 470.6 & \\
3323 & 34 & 228.51 & 8.09 & 42.95 & -21.40 & 1.22 & 399.9 & \\
3352 & 43 & 230.99 & 47.80 & 28.84 & 25.28 & 1.57 & 458.7 & \\
3431 & 34 & 234.53 & 10.74 & 48.33 & -16.14 & 1.24 & 390.5 & \\
\hline
\end{tabular}

Notes. Columns are as follows: (1) ID of the system; (2) the number of quasars in the system, $N_{\mathrm{QSO}}$; (3-4) system right ascension and declination; (5-6) system SDSS coordinates $\lambda$ and $\eta$; (7) mean redshift of the system; (8) system size; (9) comments. C1-C3: large QSO groups, described in: C1 - Clowes et al. (2013), C2, C3 - Clowes et al. (2012). 